\title{
Dinámica periódica de la repolarización como predictor de muerte súbita en pacientes con insuficiencia cardíaca crónica
}

\author{
Saúl Palacios ${ }^{1}$, Iwona Cygankiewicz ${ }^{2}$, Antoni Bayés-de-Luna ${ }^{3}$, Esther Pueyo ${ }^{1,4}$, Juan \\ Pablo Martínez ${ }^{1,4}$
}

\author{
${ }^{1}$ Biomedical Signal Interpretation and Computational Simulation (BSICoS), Instituto de Investigación en Ingeniería de \\ Aragón (I3A), IIS Aragón, Universidad de Zaragoza, Mariano Esquillor s/n, 50018, Zaragoza, Spain. \\ Tel. +34-976762707, e-mail: spalacios@unizar.es \\ ${ }^{2}$ Department of Electrocardiology, Medical University of Lodz, Lodz, Poland \\ ${ }^{3}$ Institut Català de Ciències Cardiovasculars, Santa Creu i Sant Pau Hospital, Barcelona, Spain \\ ${ }^{4}$ CIBER en Bioingeniería, Biomateriales y Nanomedicina (CIBER-BBN), Zaragoza, Spain
}

\section{Resumen}

Este trabajo evalúa la capacidad del índice Periodic Repolarization Dynamics (PRD) para predecir el riesgo de sufrir muerte súbita cardíaca (MSC) o muerte por fallo de bomba (MFB) en pacientes con insuficiencia cardíaca crónica (ICC).

\section{Introducción}

La insuficiencia cardíaca crónica (ICC) es un síndrome cardíaco que se asocia, entre otros, con un desajuste en el sistema nervioso autónomo caracterizado por un aumento de la actividad simpática y una reducción de la actividad parasimpática. La muerte súbita cardíaca (MSC) representa la principal causa de mortalidad en pacientes con ICC, siendo otra causa común de fallecimiento la muerte por fallo de bomba (MFB), que se produce cuando hay una disminución progresiva de la capacidad de bombeo del corazón.

La Dinámica Periódica de la Repolarización (en inglés “Periodic Repolarization Dynamics”, PRD) es un índice utilizado para evaluar la modulación simpática de la repolarización ventricular a través del electrocardiograma (ECG) [1]. El objetivo de este trabajo es evaluar la capacidad del índice PRD para estratificar pacientes con ICC según el riesgo de fallecer por MFB o MSC.

\section{Población de estudio}

Se analizaron registros ECG de alta resolución (1000 $\mathrm{Hz}$ ) y 20 minutos de duración en estado basal de 569 pacientes con ICC sintomática. Estos pacientes formaban parte del estudio MUSIC (Muerte Súbita en Insuficiencia Cardíaca) que fue diseñado para evaluar distintos predictores de riesgo de muerte cardíaca y MSC en pacientes ambulatorios con ICC [2]. Se realizó un seguimiento clínico a los pacientes cada 6 meses durante un promedio de 48 meses.

\section{Métodos}

El preprocesamiento de las señales de ECG consistió en la eliminación de ruido de red mediante un filtro elimina-banda a $50 \mathrm{~Hz}$, la detección y etiquetado de latidos cardíacos, un filtrado paso-bajo de $40 \mathrm{~Hz}$ y la eliminación de la línea de base mediante la interpolación con splines cúbicos.

Se definió el inicio y final de cada onda T del ECG, $\mathrm{Ton}_{\mathrm{i}} \mathrm{y} \mathrm{Toff}_{\mathrm{i}}$, respectivamente, con respecto a la posición de cada complejo QRS, $\mathrm{QRS}_{\mathrm{i}}$, considerando el correspondiente intervalo $R R\left(R_{i}\right)$, donde $i$ denota el índice del latido. Para el inicio de la ventana, $T_{o n_{i}}=Q R S_{i}+90 \mathrm{~ms}$. Para el final de la ventana, cuando $\mathrm{RR}_{\mathrm{i}}<720 \mathrm{~ms}, T_{\text {off }}=Q R S_{i}+$ $\min \left(360, \frac{2}{3} R R_{i}\right) \mathrm{ms} \mathrm{y}$, en caso de que $\mathrm{RRi} \geq 720 \mathrm{~ms}$, $T_{o f f_{i}}=Q R S_{i}+360 \mathrm{~ms}$. El ruido de cada onda $\mathrm{T}$ se estimó a partir de las componentes de alta frecuencia (por encima de $15 \mathrm{~Hz}$ ), y si el nivel de ruido $>140 \mu \mathrm{V}$ la marca QRS correspondiente se mantuvo, pero tanto Ton como Toff se descartaron.

La serie de ángulos entre ondas $\mathrm{T}$ consecutivas, $\mathrm{dT}^{\mathrm{o}}$ se calculó mediante el método descrito originalmente en [1] pero actualizado según [3]. Posteriormente, aplicando el método de Phase-Rectified Signal Averaging (PRSA) se obtuvo la serie PRSA y se definió PRD como la diferencia entre los valores máximo y mínimo de la serie de PRSA. Los ECGs se dividieron en segmentos de 5 minutos y para cada uno de ellos se calculó el valor de PRD. Se asoció al paciente el mínimo valor de PRD entre todos los segmentos calculados.

Mediante el método de Kaplan-Meier se estimó la probabilidad de supervivencia a lo largo del tiempo. Se utilizó el modelo de regresión de Cox para estimar la capacidad de predicción de MSC. Los tests con $\mathrm{p}<0,05$ se consideran significativos. Las variables con diferencias significativas en la predicción de los 
tiempos de supervivencia se utilizaron posteriormente en el modelo de regresión de Cox.

\section{Resultados}

Las distribuciones de PRD para las víctimas de MSC, las víctimas de MFB y el resto de pacientes se encuentran en los diagramas de cajas (Figura 1). Se observa que las víctimas de MSC tienen valores de PRD mayores que las víctimas de MFB (mediana [ rango intercuartílico] de 1,57 [1,21] vs 1,41 [0,91] grados) y que el resto de pacientes $(1,24$ [1,28] grados). Ninguna de las diferencias es significativa.

Tomando como umbral el valor mediano de toda la población $(1,31)$, se definieron grupos de bajo riesgo $\left(\mathrm{PRD} \leq 1,31^{\circ}\right)$ y de alto riesgo $\left(\mathrm{PRD}>1,31^{\circ}\right)$. Para MFB, no se encontró diferencia significativa entre los tiempos de supervivencia de los dos grupos. Por el contrario, para MSC, las diferencia entre los dos grupos fue estadísticamente significativa $(\mathrm{p}=0,036)$. Las curvas de probabilidad de supervivencia a MSC se muestran en la figura 2.

\section{Conclusiones}

Se ha cuantificado el marcador PRD en ECGs de pacientes con ICC. Valores elevados de PRD, representativos de una mayor modulación simpática de la repolarización, serían indicativos de un mayor riesgo de fallecer por MSC. Estos resultados concuerdan con estudios teóricos que relacionan la magnitud de oscilación de la repolarización y la generación de eventos arrítmicos [4].

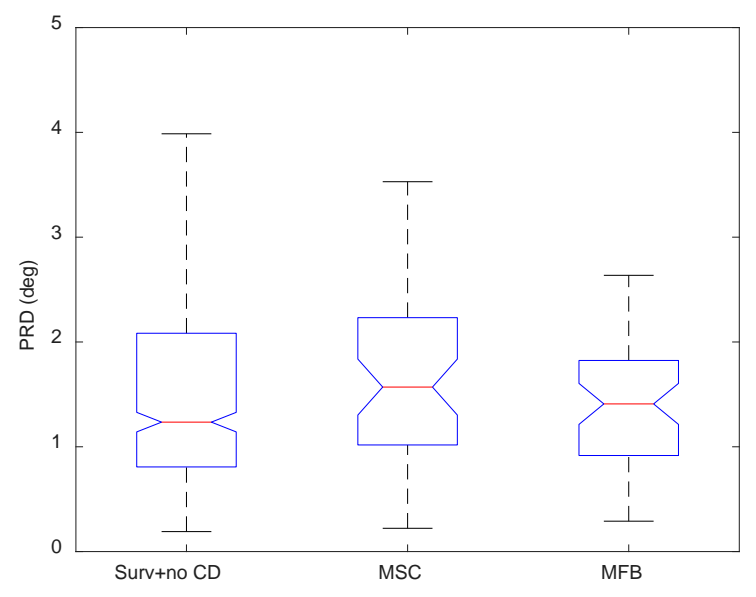

Figura 1. Diagramas de caja para tres subgrupos de pacientes: MSC, MFB y el resto de pacientes (supervivientes y víctimas de muerte no cardíaca).

\section{Agradecimientos}

Los autores agradecen el apoyo de los proyectos ERC-StG 638284 (European Research Council), PID2019-105674RB-I00 y PID2019-104881RB-I00 (Ministerio de Ciencia e Innovación) y LMP124-18 y el grupo de referencia BSICoS T39-20R (Gobierno de Aragón y FEDER 2014-2020 “Construyendo Europa desde Aragón”). Los cálculos computacionales fueron realizados en la ICTS NANBIOSIS (HPC Unit at University of Zaragoza).

\section{REFERENCIAS}

[1]. RIZAS KD ET AL. Sympathetic activity-associated periodic repolarization dynamics predict mortality following myocardial infarction. The Journal of Clinical Investigation. 2014. 124(4), 1770-80. Available from: doi: 10.1172/JCI70085.

[2]. VAZQUEZ R ET AL. The MUSIC Risk score: a simple method for predicting mortality in ambulatory patients with chronic heart failure. European Heart Journal. 2009. 30(9), 1088-1096. Available from: 10.1093/eurheartj/ehp032.

[3]. PALACIOS S ET AL. Long-term microgravity exposure increases ECG repolarization instability manifested by low-frequency oscillations of T-wave vector. Frontiers in Physiology. 2019. 10, 1510. Available from: 10.3389/fphys.2019.01510.

[4]. SAMPEDRO PUENTE DA ET AL. Mechanisms underlying interactions between low-frequency oscillations and beat-to-beat variability of celullar ventricular repolarization in response to sympathetic stimulation: Implications for arrhythmogenesis. Frontiers in Physiology. 2019. 10, 916. Available from: 10.3389/fphys.2019.00916.

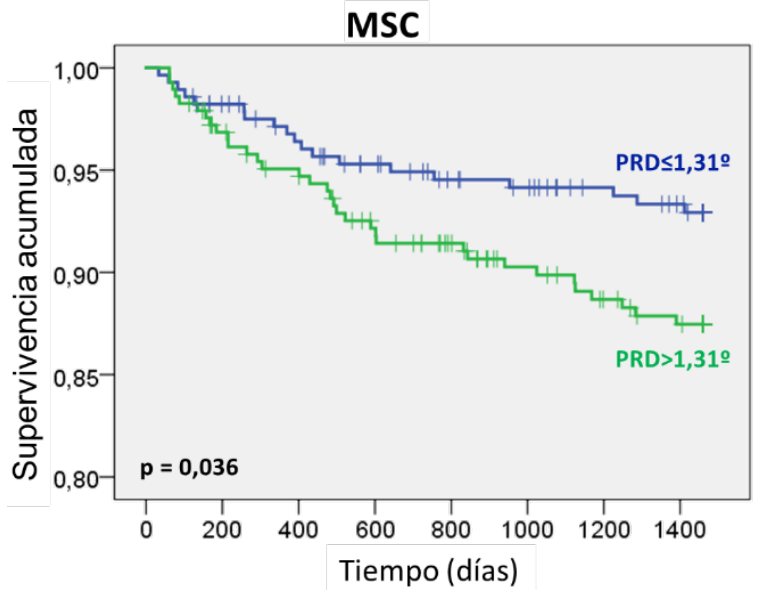

Figura 2. Curvas de Kaplan-Meier para los grupos de bajo y alto riesgo en relación a la MSC como causa de fallecimiento. 